\title{
Erratum: Interactions between copper and transition metal dichalcogenides: A density functional theory study [Phys. Rev. Materials 1, 034001 (2017)]
}

\author{
Benjamin A. Helfrecht, David M. Guzman, Nicolas Onofrio, and Alejandro H. Strachan
}

(Received 11 September 2019; published 17 October 2019)

DOI: 10.1103/PhysRevMaterials.3.109903

In the original article, the density functional theory (DFT) calculations of the conduction-band minimum (CBM) and the valence-band maximum (VBM) for $\mathrm{V} X_{2}$ only took one of the electron spins into account. If both spins are taken into account, the band alignment is modified slightly, affecting the Schottky barrier calculations. Hence, our statements that $\mathrm{VS}_{2}$ and $\mathrm{VSe}_{2}$ would form Schottky barriers with $\mathrm{Cu}(111)$ is incorrect. Interestingly, the corrected results better fit the overall trend that we see regarding Schottky barriers: The transition metal dichalcogenides (TMDs) of groups IV and V do not appear to form Schottky barriers with $\mathrm{Cu}(111)$, but the TMDs of group VI appear to form Schottky barriers roughly $0.5 \mathrm{eV}$ in height. Figures E1 and E2 are corrected versions of Figs. 10 and 11 in the original paper, and the corrected data are highlighted. Furthermore, the units of the charge density in Fig. 8(d) should also be listed as electrons/ $\AA$, instead of $\mathrm{eV} / \AA$, and the energy difference between the T and $\mathrm{T}^{\prime}$ phases of $\mathrm{VTe}_{2}$ should be listed as $17 \mathrm{meV}$ instead of $36 \mathrm{meV}$.

We also discovered typographical and spreadsheet errors in some of the tables included in the Supplemental Material [1]. The corrected data are provided in Tables E1-E4 with corrected values highlighted in red. Corrections to the cohesive energies result in root-mean-square percent errors of approximately $10 \%$ for the Perdew-Burke-Ernzerhof generalized gradient approximation (GGA-PBE) and approximately $12 \%$ for GGA-PBE with the DFT-D3 van der Waals correction against the available experimental data. The changes are minor and do not affect our results or conclusions.

The $k$-grid sizes listed in the Assessment of Calculation Accuracy section of the Supplemental Material [1] should be listed as $18 \times 18 \times 4$ and $18 \times 18 \times 8$ for the $2 \mathrm{H}$ and $1 \mathrm{~T}$ phases, respectively. However, the $k$-grids for the GGA-PBE calculations were $18 \times 18 \times 4$ for both the $2 \mathrm{H}$ and the $1 \mathrm{~T}$ phases.

Finally, the atomic structures in Figs. 6, 8(c), and S2 in the Supplemental Material [1] were rendered using VESTA [2]. A citation to the program should be given, and is provided in the bibliography of this paper.

The PYTHON programming language [3,4], including the NUMPY [5,6], SCIPY [7], and ATOMIC SIMULATION ENVIRONMENT [8] packages, in addition to the VTST tools of the Henkelman Research Group [9], were used in the postprocessing of the DFT data; plots were created using MATPLOTLIB [10] or the ORIGINPRO software maintained by the OriginLab Corporation.

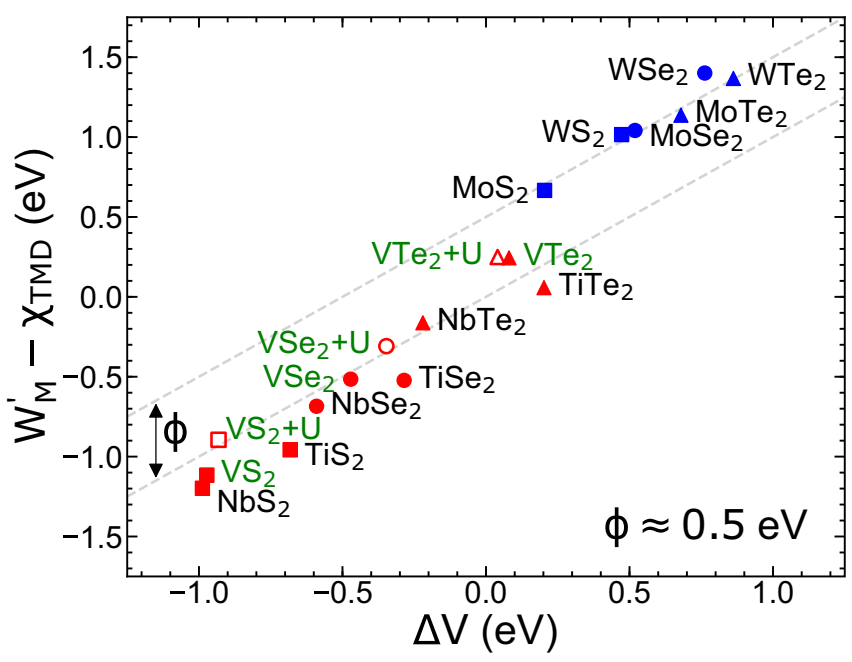

FIG. E1. Correction to Fig. 10 where the labels of the modified data are colored green: Representation of Schottky barrier formation in the $\mathrm{Cu}$ /TMD hybrids as a result of the potential step: the Schottky barrier in the semiconductor TMDs is the vertical offset $\phi$ from the lower line, which has slope 1 . Note that $\chi_{\mathrm{TMD}}$ for the metallic TMDs is simply the Fermi level. Open symbols indicate DFT $+U$ results. The phase of each TMD is that corresponding to the favored freestanding structure as shown in Fig. 7. 

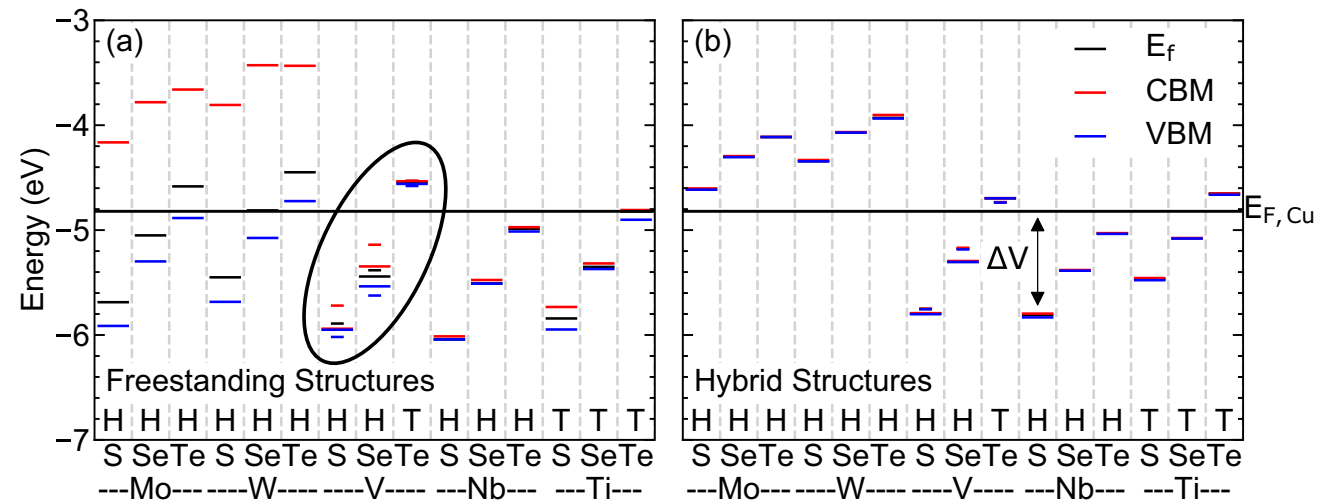

FIG. E2. Correction to Fig. 11 where the modified data are circled: Band alignment for (a) freestanding TMDs in the ground-state phase and (b) the hybrid $\mathrm{Cu}(111) / \mathrm{TMD}$ slabs with the Fermi energy of a pure copper slab $E_{F, \mathrm{Cu}}$, long horizontal black line for comparison. The Fermi level $E_{F}, \mathrm{CBM}$, and VBM are given for each freestanding and hybrid structure as the short black, red, and blue horizontal lines. Half-length lines indicate the Fermi level, CBM, and VBM obtained from DFT $+U$ for $\mathrm{VX}_{2}$. The phase of the TMD is indicated by $\mathrm{H}$ or T.

TABLE E1. Correction to Table S2 with changes in red: Lattice matching parameters $\left(n_{1}, n_{2}, m_{1}, m_{2}\right)$, supercell size, number of TMD formula units $(F)$, total number of atoms in the supercell $(N)$, supercell parameter $(a)$, and the strain on the Cu slab for the two lowest-strain orientations of the $15 \mathrm{H}$ phase TMDs. Negative strains indicate compression of the $\mathrm{Cu}$ slab; positive strains indicate tension.

\begin{tabular}{|c|c|c|c|c|c|c|c|c|c|c|c|c|}
\hline \multirow[b]{2}{*}{ TMD } & \multicolumn{6}{|c|}{ Lowest strain orientation } & \multicolumn{6}{|c|}{ Second lowest strain orientation } \\
\hline & $\begin{array}{l}n_{1}, n_{2} \\
m_{1}, m_{2}\end{array}$ & $\begin{array}{l}\text { TMD supercell } \\
\mathrm{Cu} \text { supercell }\end{array}$ & $F$ & $N$ & $a(\AA)$ & $\begin{array}{c}\mathrm{Cu} \\
\text { strain }(\%)\end{array}$ & $\begin{array}{l}n_{1}, n_{2} \\
m_{1}, m_{2}\end{array}$ & $\begin{array}{l}\text { TMD supercell } \\
\text { Cu supercell }\end{array}$ & $F$ & $N$ & $a(\AA)$ & $\begin{array}{c}\mathrm{Cu} \\
\text { strain }(\%)\end{array}$ \\
\hline $\mathrm{MoS}_{2}$ & $2,2,2,3$ & $\begin{array}{l}\sqrt{12} \times \sqrt{12} \\
\sqrt{19} \times \sqrt{19}\end{array}$ & 12 & 150 & 10.96 & -0.32 & $0,4,5,0$ & $\begin{array}{l}4 \times 4 \\
5 \times 5\end{array}$ & 16 & 198 & 12.65 & +0.35 \\
\hline $\mathrm{MoSe}_{2}$ & $1,2,2,2$ & $\begin{array}{c}\sqrt{7} \times \sqrt{7} \\
\sqrt{12} \times \sqrt{12}\end{array}$ & 7 & 93 & 8.72 & -0.17 & $2,0,1,2$ & $\begin{array}{c}2 \times 2 \\
\sqrt{7} \times \sqrt{7}\end{array}$ & 4 & 54 & 6.59 & -1.20 \\
\hline $\mathrm{MoTe}_{2}$ & $3,1,0,5$ & $\begin{array}{c}\sqrt{13} \times \sqrt{13} \\
5 \times 5\end{array}$ & 13 & 189 & 12.69 & +0.67 & $1,2,3,1$ & $\begin{array}{c}\sqrt{7} \times \sqrt{7} \\
\sqrt{13} \times \sqrt{13}\end{array}$ & 7 & 99 & 9.31 & +2.44 \\
\hline $\mathrm{WS}_{2}$ & $2,2,2,3$ & $\begin{array}{l}\sqrt{12} \times \sqrt{12} \\
\sqrt{19} \times \sqrt{19}\end{array}$ & 12 & 150 & 10.98 & -0.13 & $0,4,5,0$ & $\begin{array}{l}4 \times 4 \\
5 \times 5\end{array}$ & 16 & 198 & 12.68 & +0.54 \\
\hline $\mathrm{WSe}_{2}$ & $2,1,2,2$ & $\begin{array}{c}\sqrt{7} \times \sqrt{7} \\
\sqrt{12} \times \sqrt{12}\end{array}$ & 7 & 93 & 8.70 & -0.38 & $2,0,1,2$ & $\begin{array}{c}2 \times 2 \\
\sqrt{7} \times \sqrt{7}\end{array}$ & 4 & 54 & 6.58 & -1.40 \\
\hline $\mathrm{WTe}_{2}$ & $1,3,0,5$ & $\begin{array}{c}\sqrt{13} \times \sqrt{13} \\
5 \times 5\end{array}$ & 13 & 189 & 12.68 & +0.56 & $1,2,3,1$ & $\begin{aligned} \sqrt{7} & \times \sqrt{7} \\
\sqrt{13} & \times \sqrt{13}\end{aligned}$ & 7 & 99 & 9.30 & +2.33 \\
\hline $\mathrm{NbS}_{2}$ & $0,2,2,1$ & $\begin{array}{c}2 \times 2 \\
\sqrt{7} \times \sqrt{7}\end{array}$ & 4 & 54 & 6.68 & +0.11 & $3,0,4,0$ & $\begin{array}{l}3 \times 3 \\
4 \times 4\end{array}$ & 9 & 123 & 10.02 & -0.68 \\
\hline $\mathrm{NbSe}_{2}$ & $1,2,1,3$ & $\begin{aligned} \sqrt{7} & \times \sqrt{7} \\
\sqrt{13} & \times \sqrt{13}\end{aligned}$ & 7 & 99 & 9.16 & +0.74 & $1,3,5,0$ & $\begin{aligned} \sqrt{13} & \times \sqrt{13} \\
5 & \times 5\end{aligned}$ & 13 & 189 & 12.48 & -1.00 \\
\hline $\mathrm{NbTe}_{2}$ & $2,2,0,5$ & $\begin{array}{c}\sqrt{12} \times \sqrt{12} \\
5 \times 5\end{array}$ & 12 & 186 & 12.64 & +0.28 & $3,0,2,3$ & $\begin{array}{l}\sqrt{19} \times \sqrt{19} \\
\sqrt{19} \times \sqrt{19}\end{array}$ & 9 & 141 & 10.95 & -0.38 \\
\hline $\mathrm{TiS}_{2}$ & $0,2,1,2$ & $\begin{array}{c}2 \times 2 \\
\sqrt{7} \times \sqrt{7}\end{array}$ & 4 & 54 & 6.66 & -0.13 & $2,1,2,2$ & $\begin{array}{c}\sqrt{7} \times \sqrt{7} \\
\sqrt{12} \times \sqrt{12}\end{array}$ & 7 & 93 & 8.81 & +0.91 \\
\hline $\mathrm{TiSe}_{2}$ & $1,3,0,5$ & $\begin{aligned} \sqrt{13} & \times \sqrt{13} \\
5 & \times 5\end{aligned}$ & 13 & 189 & 12.55 & -0.45 & $1,2,3,1$ & $\begin{aligned} \sqrt{7} & \times \sqrt{7} \\
\sqrt{13} & \times \sqrt{13}\end{aligned}$ & 7 & 99 & 9.21 & +1.30 \\
\hline $\mathrm{TiTe}_{2}$ & $0,3,3,2$ & $\begin{aligned} 3 & \times 3 \\
\sqrt{19} & \times \sqrt{19}\end{aligned}$ & 9 & 141 & 11.16 & +1.52 & $2,0,3,0$ & $\begin{array}{l}2 \times 2 \\
3 \times 3\end{array}$ & 4 & 66 & 7.44 & -1.66 \\
\hline $\mathrm{VS}_{2}$ & $2,2,2,3$ & $\begin{array}{l}\sqrt{12} \times \sqrt{12} \\
\sqrt{19} \times \sqrt{19}\end{array}$ & 12 & 150 & 10.97 & -0.19 & $0,4,5,0$ & $\begin{array}{l}4 \times 4 \\
5 \times 5\end{array}$ & 16 & 198 & 12.67 & +0.47 \\
\hline $\mathrm{VSe}_{2}$ & $0,2,1,2$ & $\begin{array}{c}2 \times 2 \\
\sqrt{7} \times \sqrt{7}\end{array}$ & 4 & 54 & 6.65 & -0.34 & $2,1,2,2$ & $\begin{aligned} \sqrt{7} & \times \sqrt{7} \\
\sqrt{12} & \times \sqrt{12}\end{aligned}$ & 7 & 93 & 8.80 & +0.69 \\
\hline $\mathrm{VTe}_{2}$ & $1,3,0,5$ & $\begin{aligned} \sqrt{13} & \times \sqrt{13} \\
5 & \times 5\end{aligned}$ & 13 & 189 & 12.84 & +1.80 & $2,2,5,0$ & $\begin{aligned} \sqrt{12} & \times \sqrt{12} \\
5 & \times 5\end{aligned}$ & 12 & 186 & 12.33 & -2.19 \\
\hline
\end{tabular}


TABLE E2. Correction to Table S3 with changes in red: Lattice matching parameters $\left(n_{1}, n_{2}, m_{1}, m_{2}\right)$, supercell size, number of TMD formula units $(F)$, total number of atoms in the supercell $(N)$, supercell parameter $(a)$, and strain on the Cu slab for the two lowest-strain orientations of the $15 \mathrm{~T}$ phase TMDs. Negative strains indicate compression of the $\mathrm{Cu}$ slab; positive strains indicate tension.

\begin{tabular}{|c|c|c|c|c|c|c|c|c|c|c|c|c|}
\hline \multirow[b]{2}{*}{ TMD } & \multicolumn{6}{|c|}{ Lowest strain orientation } & \multicolumn{6}{|c|}{ Second lowest strain orientation } \\
\hline & $\begin{array}{l}n_{1}, n_{2} \\
m_{1}, m_{2}\end{array}$ & $\begin{array}{l}\text { TMD supercell } \\
\mathrm{Cu} \text { supercell }\end{array}$ & $F$ & $N$ & $a(\AA)$ & $\begin{array}{c}\mathrm{Cu} \\
\text { strain }(\%)\end{array}$ & $\begin{array}{l}n_{1}, n_{2} \\
m_{1}, m_{2}\end{array}$ & $\begin{array}{l}\text { TMD supercell } \\
\mathrm{Cu} \text { supercell }\end{array}$ & $F$ & $N$ & $a(\AA)$ & $\begin{array}{c}\mathrm{Cu} \\
\text { strain }(\%)\end{array}$ \\
\hline $\mathrm{MoS}_{2}$ & $0,4,0,5$ & $\begin{array}{l}4 \times 4 \\
5 \times 5\end{array}$ & 16 & 198 & 12.59 & -0.11 & $2,2,3,2$ & $\begin{array}{l}\sqrt{12} \times \sqrt{12} \\
\sqrt{19} \times \sqrt{19}\end{array}$ & 12 & 150 & 10.91 & -0.77 \\
\hline $\mathrm{MoSe}_{2}$ & $1,3,4,1$ & $\begin{array}{l}\sqrt{13} \times \sqrt{13} \\
\sqrt{21} \times \sqrt{21}\end{array}$ & 13 & 165 & 11.72 & +1.43 & $2,1,2,2$ & $\begin{array}{c}\sqrt{7} \times \sqrt{7} \\
\sqrt{12} \times \sqrt{12}\end{array}$ & 7 & 93 & 8.6 & -1.54 \\
\hline $\mathrm{MoTe}_{2}$ & $1,2,1,3$ & $\begin{array}{c}\sqrt{7} \times \sqrt{7} \\
\sqrt{13} \times \sqrt{13}\end{array}$ & 7 & 99 & 9.15 & +0.62 & $1,3,5,0$ & $\begin{aligned} \sqrt{13} & \times \sqrt{13} \\
5 & \times 5\end{aligned}$ & 13 & 189 & 12.47 & -1.12 \\
\hline $\mathrm{WS}_{2}$ & $2,2,3,2$ & $\begin{array}{l}\sqrt{12} \times \sqrt{12} \\
\sqrt{19} \times \sqrt{19}\end{array}$ & 12 & 150 & 11.01 & +0.20 & $3,1,4,1$ & $\begin{array}{l}\sqrt{13} \times \sqrt{13} \\
\sqrt{21} \times \sqrt{21}\end{array}$ & 13 & 165 & 11.46 & -0.80 \\
\hline $\mathrm{WSe}_{2}$ & $1,3,4,1$ & $\begin{array}{l}\sqrt{13} \times \sqrt{13} \\
\sqrt{21} \times \sqrt{21}\end{array}$ & 13 & 165 & 11.70 & +1.28 & $2,1,2,2$ & $\begin{array}{c}\sqrt{7} \times \sqrt{7} \\
\sqrt{12} \times \sqrt{12}\end{array}$ & 7 & 93 & 8.59 & -1.69 \\
\hline $\mathrm{WTe}_{2}$ & $1,2,1,3$ & $\begin{aligned} \sqrt{7} & \times \sqrt{7} \\
\sqrt{13} & \times \sqrt{13}\end{aligned}$ & 7 & 99 & 9.15 & +0.65 & $1,3,5,0$ & $\begin{array}{c}\sqrt{13} \times \sqrt{13} \\
5 \times 5\end{array}$ & 13 & 189 & 12.47 & -1.09 \\
\hline $\mathrm{NbS}_{2}$ & $0,3,0,4$ & $\begin{array}{l}3 \times 3 \\
4 \times 4\end{array}$ & 9 & 123 & 10.09 & $<+0.01$ & $0,2,1,2$ & $\begin{array}{c}2 \times 2 \\
\sqrt{7} \times \sqrt{7}\end{array}$ & 4 & 54 & 6.73 & +0.80 \\
\hline $\mathrm{NbSe}_{2}$ & $1,2,1,3$ & $\begin{aligned} \sqrt{7} & \times \sqrt{7} \\
\sqrt{13} & \times \sqrt{13}\end{aligned}$ & 7 & 99 & 9.16 & +0.79 & $1,3,5,0$ & $\begin{aligned} \sqrt{13} & \times \sqrt{13} \\
5 & \times 5\end{aligned}$ & 13 & 189 & 12.49 & -0.95 \\
\hline $\mathrm{NbTe}_{2}$ & $2,2,0,5$ & $\begin{array}{c}\sqrt{12} \times \sqrt{12} \\
5 \times 5\end{array}$ & 12 & 186 & 12.55 & -0.43 & $3,0,2,3$ & $\begin{array}{c}3 \times 3 \\
\sqrt{19} \times \sqrt{19}\end{array}$ & 9 & 141 & 10.87 & -1.09 \\
\hline $\mathrm{TiS}_{2}$ & $0,3,0,4$ & $\begin{array}{l}3 \times 3 \\
4 \times 4\end{array}$ & 9 & 123 & 10.19 & +1.05 & $2,1,3,1$ & $\begin{aligned} \sqrt{7} & \times \sqrt{7} \\
\sqrt{13} & \times \sqrt{13}\end{aligned}$ & 7 & 99 & 8.99 & -1.13 \\
\hline $\mathrm{TiSe}_{2}$ & $1,3,0,5$ & $\begin{aligned} \sqrt{13} & \times \sqrt{13} \\
5 & \times 5\end{aligned}$ & 13 & 189 & 12.70 & +0.73 & $1,2,3,1$ & $\begin{aligned} \sqrt{7} & \times \sqrt{7} \\
\sqrt{13} & \times \sqrt{13}\end{aligned}$ & 7 & 99 & 9.32 & +2.50 \\
\hline $\mathrm{TiTe}_{2}$ & $0,2,0,3$ & $\begin{array}{l}2 \times 2 \\
3 \times 3\end{array}$ & 4 & 66 & 7.46 & -1.41 & $3,0,3,2$ & $\begin{aligned} 3 & \times 3 \\
\sqrt{19} & \times \sqrt{19}\end{aligned}$ & 9 & 141 & 11.19 & +1.79 \\
\hline $\mathrm{VS}_{2}$ & $2,2,2,3$ & $\begin{array}{l}\sqrt{12} \times \sqrt{12} \\
\sqrt{19} \times \sqrt{19}\end{array}$ & 12 & 150 & 10.98 & -0.13 & $0,4,5,0$ & $\begin{array}{l}4 \times 4 \\
5 \times 5\end{array}$ & 16 & 198 & 12.68 & +0.54 \\
\hline $\mathrm{VSe}_{2}$ & $0,2,1,2$ & $2 \times 2$ & 4 & 54 & 6.64 & -0.44 & $2,1,2,2$ & $\begin{array}{c}\sqrt{7} \times \sqrt{7} \\
\sqrt{12} \times \sqrt{12}\end{array}$ & 7 & 93 & 8.79 & +0.59 \\
\hline $\mathrm{VTe}_{2}$ & $1,3,0,5$ & $\begin{aligned} \sqrt{13} & \times \sqrt{13} \\
5 & \times 5\end{aligned}$ & 13 & 189 & 12.75 & +1.15 & $2,2,5,0$ & $\begin{aligned} \sqrt{12} & \times \sqrt{12} \\
5 & \times 5\end{aligned}$ & 12 & 186 & 12.25 & -2.82 \\
\hline
\end{tabular}


TABLE E3. Correction to Table S5 with changes in red: Bulk TMD cohesive energies per formula unit compared to the experimental enthalpy of formation.

\begin{tabular}{|c|c|c|c|c|c|c|}
\hline \multirow[b]{2}{*}{ TMD } & \multicolumn{3}{|c|}{ Bulk cohesive energy $\left(\mathrm{eV} / \mathrm{MX}_{2}\right)$} & \multicolumn{2}{|c|}{$\%$ Difference } & \multirow[b]{2}{*}{ Expt. Ref. } \\
\hline & DFT-D3 & GGA-PBE & Expt. & DFT-D3 & GGA-PBE & \\
\hline $2 \mathrm{H}-\mathrm{MoS}_{2}$ & -2.64 & -2.66 & -2.80 & -5.64 & -4.91 & [11] \\
\hline $2 \mathrm{H}-\mathrm{MoSe}_{2}$ & -2.03 & -2.05 & -2.40 & -15.39 & -14.73 & {$[12],[13]$} \\
\hline $2 \mathrm{H}-\mathrm{MoTe}_{2}$ & -0.70 & -0.80 & & & & \\
\hline $2 \mathrm{H}-\mathrm{WS}_{2}$ & -2.30 & -2.45 & -2.50 & -8.03 & -2.11 & [14] \\
\hline $2 \mathrm{H}-\mathrm{WSe}{ }_{2}$ & -1.62 & -1.65 & -1.90 & -14.53 & -12.97 & [11] \\
\hline $2 \mathrm{H}-\mathrm{WTe}{ }_{2}$ & -0.05 & -0.18 & & & & \\
\hline $2 \mathrm{H}-\mathrm{NbS}_{2}$ & -3.06 & -3.07 & & & & \\
\hline $2 \mathrm{H}-\mathrm{NbSe}_{2}$ & -2.56 & -2.56 & & & & \\
\hline $2 \mathrm{H}-\mathrm{NbTe}_{2}$ & -1.26 & -1.34 & & & & \\
\hline $2 \mathrm{H}-\mathrm{TiS}_{2}$ & -3.34 & -3.41 & & & & \\
\hline $2 \mathrm{H}-\mathrm{TiSe}_{2}$ & -2.92 & -2.99 & & & & \\
\hline $2 \mathrm{H}-\mathrm{TiTe}_{2}$ & -1.64 & -1.81 & & & & \\
\hline $2 \mathrm{H}-\mathrm{VS}_{2}$ & -2.47 & -2.61 & & & & \\
\hline $2 \mathrm{H}-\mathrm{VS}_{2}{ }^{\mathrm{a}}$ & -2.54 & -2.68 & & & & \\
\hline $2 \mathrm{H}-\mathrm{VSe}_{2}$ & -1.96 & -2.12 & & & & \\
\hline $2 \mathrm{H}-\mathrm{VSe}_{2}{ }^{\mathrm{a}}$ & -2.06 & -2.22 & & & & \\
\hline $2 \mathrm{H}-\mathrm{VTe}_{2}$ & -0.76 & -0.84 & & & & \\
\hline $2 \mathrm{H}-\mathrm{VTe}_{2}{ }^{\mathrm{a}}$ & -0.86 & -1.00 & & & & \\
\hline $1 \mathrm{~T}-\mathrm{MoS}_{2}$ & -1.83 & -1.82 & & & & \\
\hline $1 \mathrm{~T}-\mathrm{MoSe}_{2}$ & -1.35 & -1.34 & & & & \\
\hline $1 \mathrm{~T}-\mathrm{MoTe}_{2}$ & -0.18 & -0.28 & & & & \\
\hline $1 \mathrm{~T}-\mathrm{WS}_{2}$ & -1.42 & -1.56 & & & & \\
\hline $1 \mathrm{~T}-\mathrm{WSe}{ }_{2}$ & -0.87 & -0.88 & & & & \\
\hline $1 \mathrm{~T}-\mathrm{WTe}_{2}$ & 0.52 & 0.39 & & & & \\
\hline $1 \mathrm{~T}-\mathrm{NbS}_{2}$ & -2.95 & -2.96 & & & & \\
\hline $1 \mathrm{~T}-\mathrm{NbSe}_{2}$ & -1.43 & -2.46 & & & & \\
\hline $1 \mathrm{~T}-\mathrm{NbTe}_{2}$ & -1.19 & -1.28 & & & & \\
\hline $1 \mathrm{~T}-\mathrm{TiS}_{2}$ & -3.76 & -3.84 & & & & \\
\hline $1 \mathrm{~T}-\mathrm{TiSe}_{2}$ & -3.27 & -3.35 & & & & \\
\hline $1 \mathrm{~T}-\mathrm{TiTe}_{2}$ & -1.92 & -2.09 & & & & \\
\hline $1 \mathrm{~T}-\mathrm{VS}_{2}$ & -2.44 & -2.57 & & & & \\
\hline $1 \mathrm{~T}-\mathrm{VS}_{2}{ }^{\mathrm{a}}$ & -2.49 & -2.62 & & & & \\
\hline $1 \mathrm{~T}-\mathrm{VSe}_{2}$ & -1.94 & -2.09 & & & & \\
\hline $1 \mathrm{~T}-\mathrm{VSe}_{2}{ }^{\mathrm{a}}$ & -2.04 & -2.19 & & & & \\
\hline $1 \mathrm{~T}-\mathrm{VTe} \mathrm{T}_{2}$ & -0.79 & -0.92 & & & & \\
\hline $1 \mathrm{~T}-\mathrm{VTe}_{2}{ }^{\mathrm{a}}$ & -0.94 & -1.08 & & & & \\
\hline
\end{tabular}

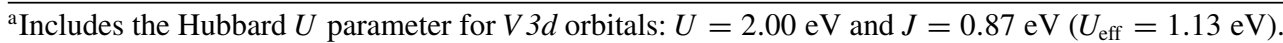

TABLE E4. Correction to Table S6 with changes in red: Comparison of binding energy $\left(E_{b}\right)$, equilibrium separation distance $(d$, between $\mathrm{Cu}$ surface and bottom chalcogen atoms in the first TMD layer), Schottky barrier $(\phi)$, and potential step $(\Delta V)$ for monolayer and bilayer $\mathrm{H}-\mathrm{MoSe}_{2}$ and $\mathrm{H}-\mathrm{NbS}_{2}$ adsorbed on $\mathrm{Cu}(111)$.

\begin{tabular}{|c|c|c|c|c|c|c|c|c|}
\hline & \multicolumn{4}{|c|}{$\mathrm{MoSe}_{2}$} & \multicolumn{4}{|c|}{$\mathrm{NbS}_{2}$} \\
\hline & $d(\AA)$ & $E_{b}\left(\mathrm{~J} / \mathrm{m}^{2}\right)$ & $\Delta V(\mathrm{eV})$ & $\phi(\mathrm{eV})$ & $d(\AA)$ & $E_{b}\left(\mathrm{~J} / \mathrm{m}^{2}\right)$ & $\Delta V(\mathrm{eV})$ & $\phi(\mathrm{eV})$ \\
\hline Monolayer & 2.39 & 1.50 & 0.52 & 0.52 & 2.05 & 2.33 & -0.99 & \\
\hline Bilayer & 2.39 & 1.54 & 0.46 & 0.45 & 2.06 & 2.39 & -1.19 & \\
\hline
\end{tabular}


[1] See Supplemental Material at http://link.aps.org/supplemental/ 10.1103/PhysRevMaterials.1.034001 for a comparison of bulk TMD lattice parameters with experiment, tabulated $\mathrm{Cu} / \mathrm{TMD}$ interface orientations and strain, and a brief examination of $\mathrm{Cu}(111)$ interfaces with multilayer TMDs.

[2] K. Momma and F. Izumi, VESTA 3 for three-dimensional visualization of crystal, volumetric, and morphology data, J. Appl. Crystallogr. 44, 1272 (2011).

[3] T. E. Oliphant, Python for scientific computing, Comput. Sci. Eng. 9, 10 (2007).

[4] K. J. Millman and M. Aivazis, Python for scientists and engineers, Comput. Sci. Eng. 13, 9 (2011).

[5] T. E. Oliphant, A Guide to NumPy (Trelgol, USA, 2006).

[6] S. van der Walt, S. C. Colbert, and G. Varoquaux, The NumPy array: A structure for efficient numerical computation, Comput. Sci. Eng. 13, 22 (2011).

[7] E. Jones, T. Oliphant, P. Peterson et al., SciPy: Open Source Scientific Tools for Python, 2001, http://www.scipy.org.

[8] A. H. Larsen, J. J. Mortensen, J. Blomqvist, I. E. Castelli, R. Christensen, M. Dułak, J. Friis, M. N. Groves, B. Hammer, C. Hargus, E. D. Hermes, P. C. Jennings, P. B. Jensen, J. Kermode, J. R. Kitchin, E. L. Kolsbjerg, J. Kubal, K. Kaasbjerg, S. Lysgaard, J. B. Maronsson, T. Maxson, T. Olsen, L. Pastewka, A. Peterson, C. Rostgaard, J. Schiøtz, O. Schütt, M. Strange, K. S. Thygesen, T. Vegge, L. Vilhelmsen, M. Walter, Z. Zeng, and $\mathrm{K}$. W. Jacobsen, The atomic simulation environment-a
Python library for working with atoms, J. Phys.: Condens. Matter 29, 273002 (2017).

[9] Henkelman Research Group, University of Texas at Austin, VTST scripts, http://theory.cm.utexas.edu/vtsttools/scripts. html.

[10] J. D. Hunter, Matplotlib: A 2D graphics environment, Comput. Sci. Eng. 9, 90 (2007).

[11] P. O'Hare, B. M. Lewis, and B. A. Parkinson, Standard molar enthalpy of formation by fluorine-combustion calorimetry of tungsten diselenide ( $\mathrm{WSe}_{2}$ ), thermodynamics of the hightemperature vaporization of $\mathrm{WSe}_{2}$, revised value of the standard molar enthalpy of formation of molybdenite $\left(\mathrm{MoS}_{2}\right)$, J. Chem. Thermodyn. 20, 681 (1988).

[12] O. Lehtinen, H.-P. Komsa, A. Pulkin, M. B. Whitwick, M.-W. Chen, T. Lehnert, M. J. Mohn, O. V. Yazyev, A. Kis, U. Kaiser, and A. V. Krasheninnikov, Atomic scale microstructure and properties of Se-deficient two-dimensional $\mathrm{MoSe}_{2}$, ACS Nano 9, 3274 (2015).

[13] P. O'Hare, I. R. Tasker, and J. M. Tarascon, A fluorinecombusion calorimetric study of two molybdenum selenides: $\mathrm{MoSe}_{2}$ and $\mathrm{Mo}_{6} \mathrm{Se}_{6}$, J. Chem. Thermodyn. 19, 61 (1987).

[14] P. O'Hare, W. N. Hubbard, G. K. Johnson, and H. E. Flowtow, Calorimetric measurements of the low-temperature heat capacity, standard molar enthalpy of formation at $298.15 \mathrm{~K}$, and hightemperature molar enthalpy increments relative to $298.15 \mathrm{~K}$ of tungsten disulphide $\left(\mathrm{WS}_{2}\right)$, and the thermodynamic properties to $1500 \mathrm{~K}$, J. Chem. Thermodyn. 16, 45 (1984). 